\title{
Improving Compliance in Remote Healthcare Systems Through Smartphone Battery Optimization
}

\author{
Nabil Alshurafa, Student Member, IEEE, Jo-Ann Eastwood, Suneil Nyamathi, Student Member, IEEE, \\ Jason J. Liu, Student Member, IEEE, Wenyao Xu, Member, IEEE, Hassan Ghasemzadeh, Member, IEEE, \\ Mohammad Pourhomayoun, Member, IEEE, and Majid Sarrafzadeh, Fellow, IEEE
}

\begin{abstract}
Remote health monitoring (RHM) has emerged as a solution to help reduce the cost burden of unhealthy lifestyles and aging populations. Enhancing compliance to prescribed medical regimens is an essential challenge to many systems, even those using smartphone technology. In this paper, we provide a technique to improve smartphone battery consumption and examine the effects of smartphone battery lifetime on compliance, in an attempt to enhance users' adherence to remote monitoring systems. We deploy WANDA-CVD, an RHM system for patients at risk of cardiovascular disease (CVD), using a wearable smartphone for detection of physical activity. We tested the battery optimization technique in an in-lab pilot study and validated its effects on compliance in the Women's Heart Health Study. The battery optimization technique enhanced the battery lifetime by $192 \%$ on average, resulting in a $53 \%$ increase in compliance in the study. A system like WANDA-CVD can help increase smartphone battery lifetime for RHM systems monitoring physical activity.
\end{abstract}

Index Terms-Battery optimization, remote health monitoring, user compliance.

\section{INTRODUCTION AND MOTIVATION}

I $\mathrm{N}$ the U.S., $75 \%$ of our health care spending focuses on chronic conditions, among which cardiovascular disease (CVD) ranks among the highest in societal burden and also the most preventable [1]. Remote health monitoring of patients with chronic heart failure has been shown to improve heart failure patients' health, resulting in reduced hospital readmissions and an overall positive effect on clinical outcomes in communities [2].

Smartphones are increasingly used in remote health monitoring (RHM) as an information gateway to extract patient information, especially physical activity (PA) [3]-[6]. They have multiple functionalities including: 1) data collector for PA from on-board sensors; 2) information hub that extracts data from other devices and user input; 3 ) data transmitter to server; and 4) feedback provider to relay information to the patient. Contin-

Manuscript received December 15, 2013; revised April 16, 2014; accepted May 26, 2014. Date of publication June 9, 2014; date of current version December 30, 2014.

N. Alshurafa, S. Nyamathi, J. Liu, M. Pourhomayoun, and M. Sarrafzadeh are with the Department of Computer Science, Wireless Health Institute, University of California, Los Angeles, CA 90095 USA (e-mail: nabil@ cs.ucla.edu).

J.-Ann Eastwood is with the School of Nursing University of California, Los Angeles CA 90095 USA (e-mail: jeastwood@ sonnet.ucla.edu).

W. Xu is with the Department of Computer Science and Engineering University at Buffalo, the State University of New York, New York, NY 14260 USA (e-mail: wenyao@cs.ucla.edu).

H. Ghasemzadeh is with the School of Electrical Engineering and Computer Science at Washington State University, Pullman, WA 99164 USA (e-mail: hassan@eecs.wsu.edu).

Color versions of one or more of the figures in this paper are available online at http://ieeexplore.ieee.org.

Digital Object Identifier 10.1109/JBHI.2014.2329712 uous data collection of PA and data transmission contribute the most to battery consumption. Patient compliance is defined as adherence to defined medical or study prescriptions. In smartphone-based RHM systems, compliance can also be affected by other factors including lack of proper education and poor mobile phone coverage (no Wi-Fi or cellular network) [3], [7], [8].

In this paper, we designed and developed a battery optimization technique and test the effects of battery lifetime of smartphones on RHM system compliance. According to Desai et al. [9], a major design challenge for remote monitoring systems is improving patient compliance and self-care. By decreasing the frequency with which a user must charge the smartphone, we potentially increase compliance with the system. This battery optimization technique has been tested both in-lab and in a clinical trial known as the Women's Heart Health study. This trial was designed to investigate the use of technology in decreasing risk factors for CVD.

This paper is organized as follows. Section II discusses related works. In Section III, we discuss the different components within the WANDA-CVD remote health monitoring system. Section IV presents our battery optimization algorithm. In Section V, we discuss the experimental setup and the clinical trial that tested the WANDA-CVD system. We, then, present our experimental results and discussion in Section VI on smartphone battery lifetime along with participant compliance rates of our WANDA-CVD system in the Women's Heart Health Study. Finally, we conclude and discuss future work in Section VII.

\section{BACKGROUND AND RELATED WORK}

Recent advances in pervasive and networking technology have enabled many remote monitoring systems [10], [11]. Despite the increase in research on RHM systems, it remains to be seen whether the technical feasibility and effectiveness of such systems will enhance patient care. Most mobile-based interventions are limited to data transfers unable to provide wireless coaching and feedback [12]. Chowdhury et al. designed MediAlly [13], a prototype that would dynamically activate the collection of data from other external sensors based on a specified context. Misra et al. designed the ACQUA [14] framework to optimize smartphone data transfer using a process of context determination. Our WANDA-CVD system, however, detects active and inactive states and adjusts the sensor sampling rate accordingly. To enhance battery life, our system also delays processing power until nurses need data, or until the smartphone is being charged. 


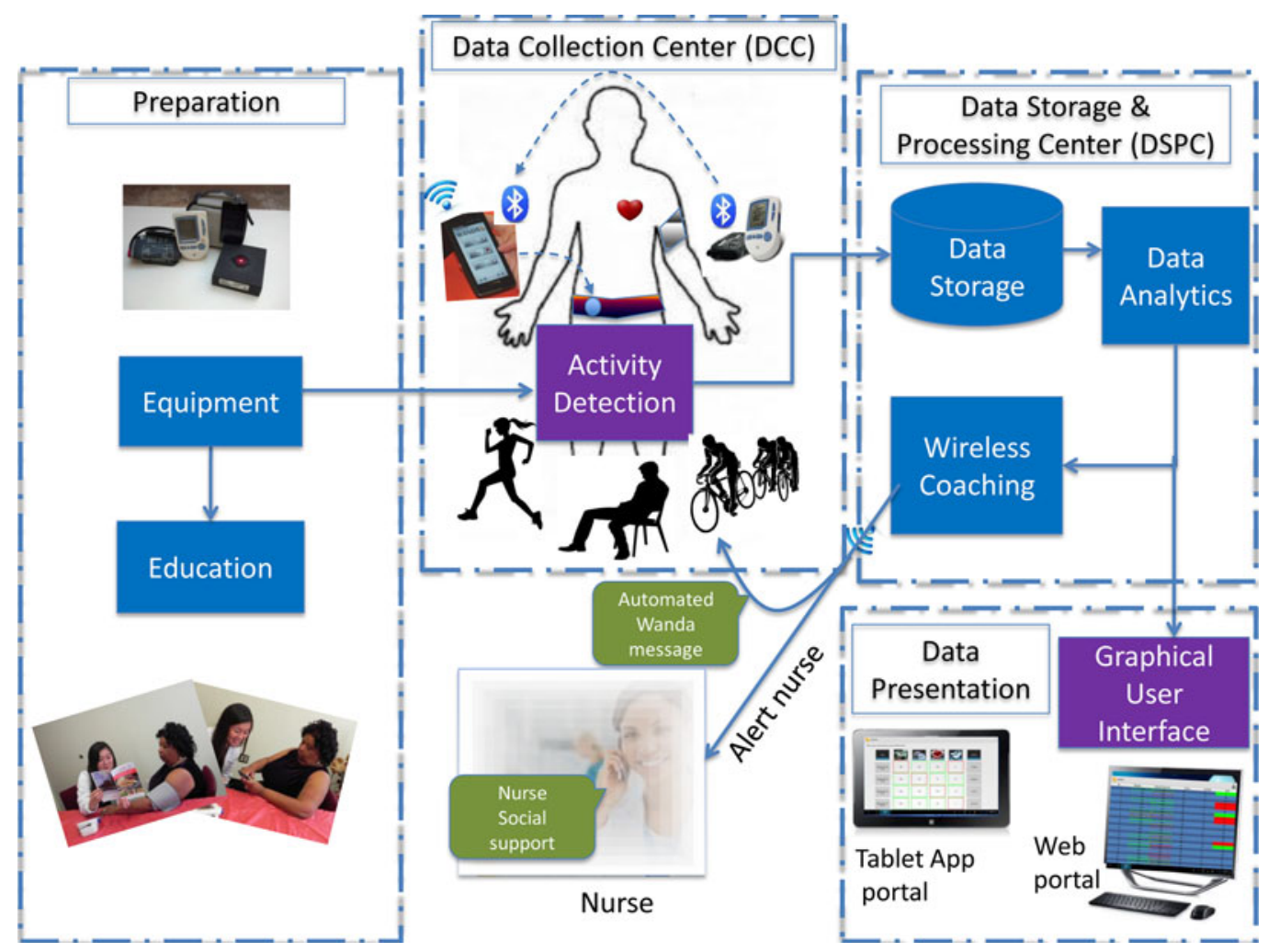

Fig. 1. WANDA-CVD system architecture comprises a DCC, a DSPC, and a medical user interface.

Many studies have performed detailed analysis of energy consumption in smartphones; Carroll et al. [15] showed how usage patterns affect overall energy consumption and battery life. In this paper, we focus on battery longevity in real world scenarios under the continuous monitoring of PA. Lee et al. [16] developed a smartphone-based mobile health monitoring system that focused on communication from a biosignal monitoring unit and measured energy expenditure with a triaxial accelerometer, but did not report on battery lifetime and compliance with the system.

Many home-based monitoring systems have involved a substantial burden on participants. In Chaudhry's telemonitoring study [17], the patient sample had been recently hospitalized with an acute exacerbation of heart failure. They were required, for six months, to make daily phone calls, record a message, and answer a series of questions. This study reported low compliance rates, and proved unsuccessful in reducing mortality in this sample of heart failure patients. Mortara et al. [18] designed a similar home monitoring system that attempted to supervise patients with heart failure and transmitted measurements once a week over slow telephone lines. This system showed little significance in reducing cardiac death or hospitalization, despite the high-reported patient compliance rate (completion of 92\% of cardiorespiratory recordings, and transmission of $81 \%$ of vital signs). WANDA-CVD attempts to tackle a more preventive approach by reducing risk factors as a preventive measure in accordance with the Institute of Medicine Report and the goals of Healthy People 2020 [19].

\section{SYSTEM ARCHITECTURE}

There are several key components in the complete design of the WANDA-CVD system illustrated in Fig. 1. The first component is the automated data collection component (DCC), which involves a smart phone application measuring, communicating, and collecting data from sensors, storing it locally, and transmitting it through a network to the data storage and processing center (DSPC). The DSPC stores the raw data collected from multiple DCC's, and deploys automated data analytics, which is then processed to determine what messages to transmit to the nurse practitioner and the participants through wireless coaching. The data presentation component displays the data for nurses to provide a visual summary of each patient's status. These components will be described in detail in this section.

\section{A. Data Collection Component (DCC)}

The DCC senses and collects data. A WANDA-CVD Android-based smartphone application has been designed to collect data from patients and transmit it to the DSPC. The application includes the following properties:

1) Smartphone interface: To appeal to the young women in our clinical trial, we selected the Motorola Droid Razr Maxx with $3300 \mathrm{mAh}$ Li-Ion battery, a lightweight smartphone with an embedded accelerometer and Bluetooth technology. The Android application is designed to run on Android version 4.0 or higher, and uses a simple and intuitive graphical user interface. 


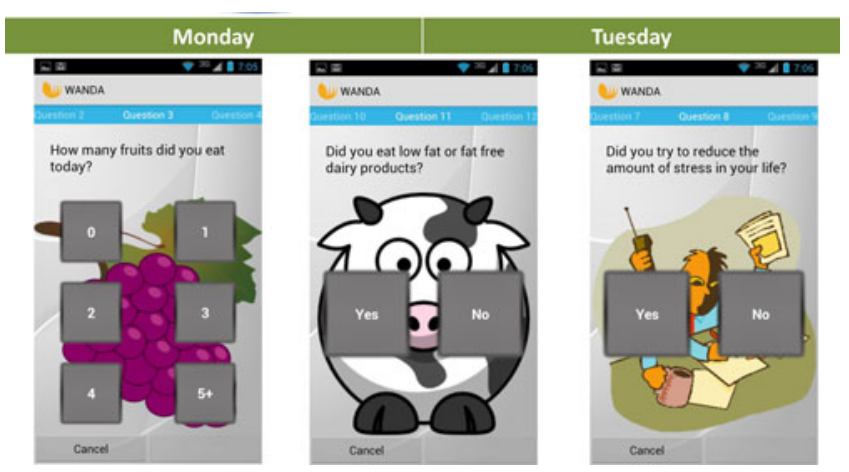

Fig. 2. Sample set of questions sent on a daily basis, to further reinforce the education received during baseline.

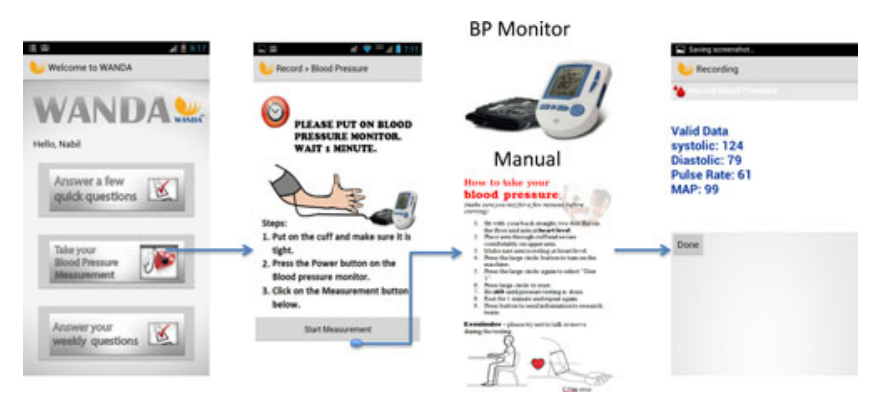

Fig. 3. Snapshot of the WANDA-CVD application showing the steps necessary for the patient to measure their BP. They simply take their BP measurement, then push a button on the application to transfer the data to the phone, which is then uploaded to the server. The participants can also see their data on the phone to validate that the data was received.

TABLE I

SMARTPHONE EVENTS AND USAGE TABLE

\begin{tabular}{lc}
\hline \hline Event Type & Description \\
\hline ACTION POWER DISCONNECTED & Phone disconnected from charge \\
ACTION POWER CONNECTED & Phone connected to charge \\
BATTERY LOW & $25 \%$ of battery remaining \\
ACTION SHUTDOWN & Battery dead and phone shutdown \\
BOOT COMPLETED & Phone turned back on \\
UPGRADED TO VERSION N & Upgraded app version number \\
\hline \hline
\end{tabular}

2) Questionnaire system:The WANDA-CVD system has several customizable questionnaire templates selected by the nurse practitioner and displayed to the participant on a daily or weekly basis. The questions relate to diet, lifestyle, and PA. Fig. 2 illustrates the different daily questionnaires (DQs) selected by the nurse prior to deployment of the Women's Heart Health study.

3) Bluetooth-enabled sensor: In the Women's Heart Health study, the nurse requested weekly monitoring of blood pressure (BP) readings from each participant. We used an FDA-approved BP monitor designed in collaboration with Fora Care [20]. Fig. 3 illustrates the participant's communication process with the BP monitor.

4) Smartphone events and usage: Nurses have the ability to observe a participant behavior regarding phone usage. As shown in Table I, when the phone is connected to or removed from a charger, runs low on battery, or is shut
TABLE II

CATEGORY COMPLIANCE DEFINITION

\begin{tabular}{lc}
\hline \hline Category (c) & Compliance Definition \\
\hline DQ & $\begin{array}{c}\text { Completed at-least 3 days out of the } \\
\text { 6 DQs a week. }\end{array}$ \\
WQ & Completed the WQ once a week. \\
BP & Measured BP atleast once a week. \\
Activity & Performed the equivalent of 20 min \\
& of low intensity PA. \\
\hline \hline
\end{tabular}

down, the event is logged. This allows the nurses to contact participants who leave their phone on the charger or allow the battery to run low, and encourage mindfulness.

\section{B. Data Storage and Processing Center (DSPC)}

The DSPC comprises two main components, namely data storage and data analytics with wireless coaching, an enhancement to our previous RHM system [21]. In order to achieve high scalability, availability, and reliability, DSPC is built using the latest Google cloud technologies [22].

After the raw data have been stored in the data storage component, data analytics are performed to identify opportunities to coach the users. The coaching is in the form of reminder messages prompting the user to take certain actions, such as taking BP measurements or enhancing exercise. The system automatically determines the appropriate message to send.

\section{Data Presentation}

Continuous nurse exposure to the data is critical. For convenience and accessibility, we have designed a system that is both web- and tablet-based. The web-based portal provides a dashboard, with a summary of all the participants' most recent information. The system provides plots over time for each measurement type so the nurse can examine trends.

\section{Compliance}

In order to quantify the feasibility of our system, we defined a metric of compliance in coordination with our nurses and medical experts. We provide an overall compliance rate, as well as a per category compliance rate. There are currently four categories requiring feedback from the participant: $\mathrm{DQ}$, weekly questionnaire (WQ), BP monitor, and physical activity (Activity). Table II describes the definition of compliance for each of the four categories. We determine compliance on a weekly basis for each category $c$, and calculate the total compliance $\mathrm{TC}_{c}$ for a specific category $c$, for all subjects $s$ (total of $m$ subjects), over $n$ weeks by

$$
\begin{aligned}
\operatorname{Com}(i)_{s}^{c} & = \begin{cases}1, & \text { if } S \text { compliant in week } i \text { in category } c \\
0, & \text { otherwise }\end{cases} \\
\operatorname{Com}_{s}^{c} & =\frac{\sum_{i=1}^{n} \operatorname{Com}(i)_{s}^{c}}{n} \\
\mathrm{TC}_{c} & =\frac{\sum_{s=1}^{m} \operatorname{Com}_{s}^{c}}{m}
\end{aligned}
$$




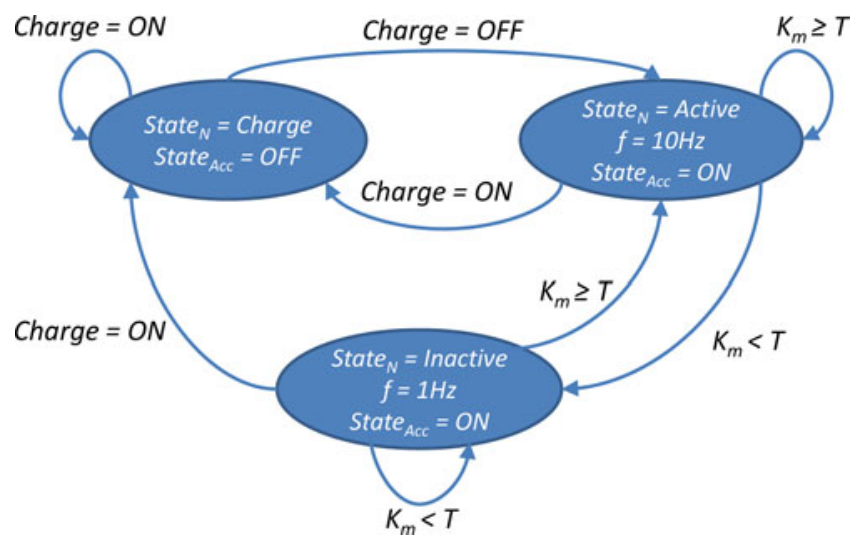

Fig. 4. Battery optimization state diagram. When the smartphone is charging it enters an initial state. Once disconnected from power, it enters an active state. If a participant is detected to have a low activity value for $60 \mathrm{~s}$, the smartphone enters an inactive state with a lower sampling rate, and once the participant becomes more active the smartphone transitions back to an active state.

An overall compliance rate is calculated and gives participants credit if they complied with at least two of the four categories.

\section{BATTERY OPTIMIZATION TECHNIQUE}

In order to maximize compliance, it is critical that the smartphone is able to last a full day of regular use. The majority of battery life is spent on processing triaxial accelerometer data and transmitting data to the servers. We initially designed the system to process the data constantly and upload it at a fixed time interval, but realizing that many individuals spend much of their days inactive, the smartphone could enter "sleep mode" to decrease the sampling rate of the accelerometer when the user is not in motion.

The battery optimization technique enters an initial state when the phone is connected to a charger. The accelerometer can be turned OFF in this state. Once the phone is disconnected from the charger, it enters an "active" state, where the accelerometer is turned $\mathrm{ON}$ and the sampling rate is set at $10 \mathrm{~Hz}$. If the user becomes stationary, as in sitting or not exerting much PA, the smartphone enters to an "inactive" state. Fig. 4 provides the state diagram that illustrates the transition from active to inactive state.

In order to determine the participants' PA level, we used the algorithm proposed by Panasonic [23] shown to have high correlation $\left(R^{2}=0.86\right)$ with Doubly Labeled Water, one of the most accurate methods for evaluating total energy expenditure under free living conditions. $K_{m}$ values, shown in (3), are calculated for a given time window and mapped to activity levels. We achieved optimal results using a $10 \mathrm{~Hz}$ sampling rate and a time window of $5 \mathrm{~s}$. Therefore, the number of samples $n$ in five s is 50. For each sample $i$, we use the data from each acceleration axis, $x_{i}, y_{i}$, and $z_{i}$, to calculate $K_{m}$ :

$$
\begin{aligned}
K_{m} & =\sqrt{\frac{1}{n-1}\left[Q-\frac{1}{n}(P)\right]} \text { where } \\
Q & =\sum_{i=0}^{n} x_{i}^{2}+\sum_{i=0}^{n} y_{i}^{2}+\sum_{i=0}^{n} z_{i}^{2} \text { and }
\end{aligned}
$$

$$
P=\left(\sum_{i=0}^{n} x_{i}\right)^{2}+\left(\sum_{i=0}^{n} y_{i}\right)^{2}+\left(\sum_{i=0}^{n} z_{i}\right)^{2} .
$$

Raw data is buffered into fixed size arrays and written to file in an efficient batch to avoid unnecessary memory allocations and garbage collection. Polling rates for accelerometer values on Android devices are hardware dependent. One minute of data are buffered before being written to file as a compromise between disk I/O and the amount of memory that needs to be reserved for such a buffer. The Android operating system terminates applications in the order of highest memory usage when resources become scarce, such as when the camera is in use or during any other computationally intensive activity. For this reason, it is imperative that the memory use of the activity collection service be as small as reasonably possible to minimize the risk of a pause in data collection. In the event that service is terminated or the phone is restarted, the data collection service will automatically reactivate.

Because our study looks at PA trends over time, we chose to accept an increase in latency when receiving data to significantly increase battery life and, thus, achieve higher compliance. By delaying until an external power source is connected, the processing and uploading of accelerometer values into meaningful data can be done without impacting battery life or generating the excess heat that can be bothersome to participants. The battery optimization technique is presented in Procedure 1. Recorded data is queued for upload in a SQLite database, which is used to maintain a synchronized state with WANDA-CVD servers. Upon connecting the device to a charger, data values are processed and uploaded completely invisibly to the end user, requiring no intervention on their part. In the case of network connectivity concerns, synchronization of all queued data will automatically occur when the phone is charged.

\section{EXPERIMENT SETUP AND ClinicAl TRIAL}

In preparation for the Women's Heart Health study, we perform an in-lab pilot study with five participants to test the smartphone application with and without battery optimization. To test effects of battery lifetime on compliance in real life, we selected seven participants from the Women's Heart Health clinical trial to test the system without battery optimization for two months and with battery optimization for the remaining four months.

The Women's Heart Health study focuses on preventive, sustainable CVD management through lifestyle changes in young black women aged 25-45 years. The study aims to determine the feasibility of using education and technology in reducing risk factors. The intervention group receives four educational classes on self-management of diet, nutrition, PA, and stress reduction. After completing baseline screening of cholesterol levels, BP, and BMI; demographic and psychosocial questionnaires; and the educational classes, the participants were taught to wear and manage the phones and BP devices. They were told that the primary purpose of the smartphone was to track their PA, while providing a user interface and a mechanism for automated feedback. The participants were asked to place the smartphone in their pocket or around their waist throughout the 


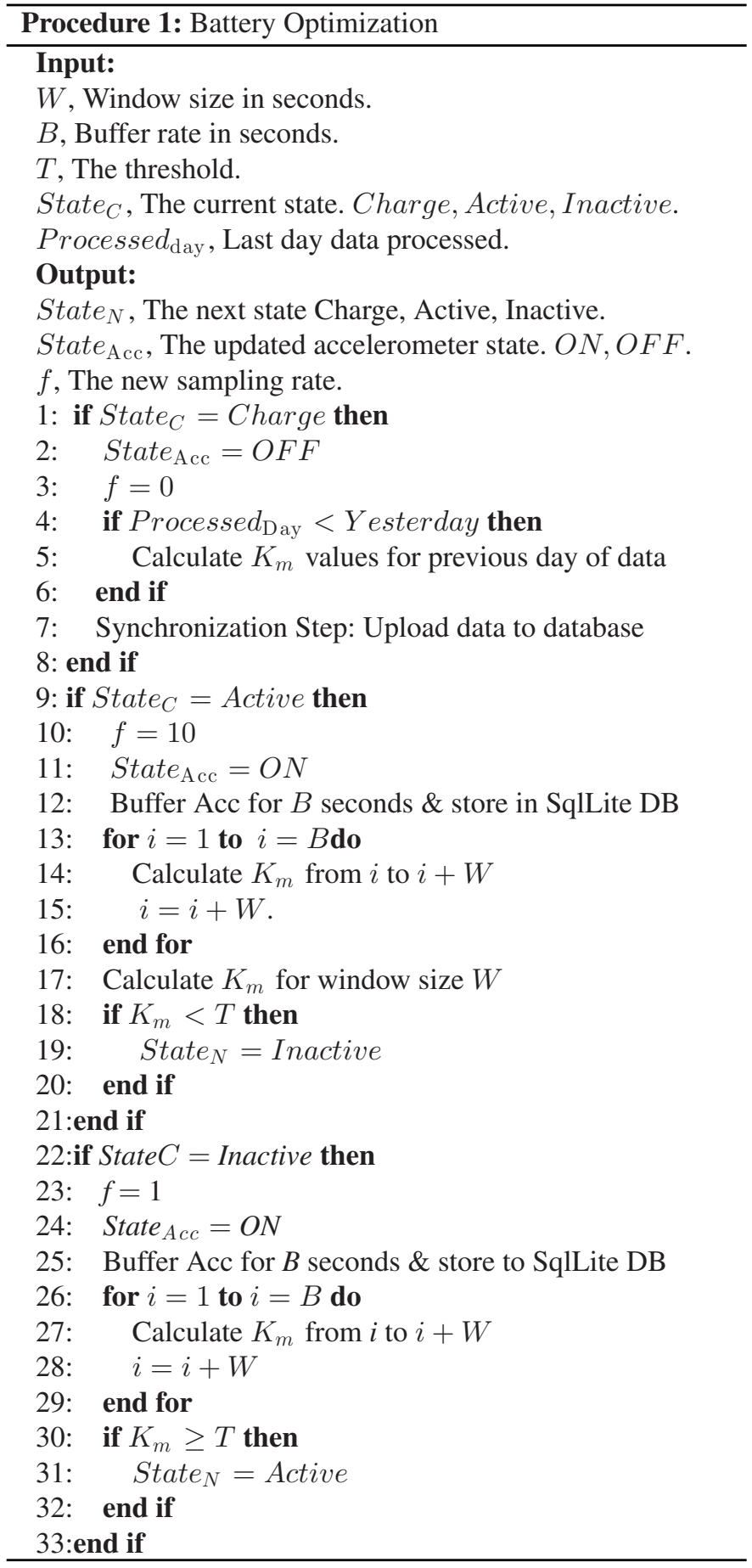

day. They are able to send/receive unlimited text messages along with unlimited data plans.

We tested the WANDA-CVD smartphone application under four configurations: 1) airplane mode, 2) Wi-Fi only, 3) NG only, and 4) Wi-Fi and NG both enabled. NG represents the cellular network coverage whose changes based on locale between $2 \mathrm{G}$, $3 \mathrm{G}$, and 4G/LTE networks.

We tested each configuration in both a geo-active and geoinactive mode. Geo-inactive mode represents the participant that uses the smartphone predominantly at home or in a single location where Wi-Fi and NG coverage exist. Geo-active

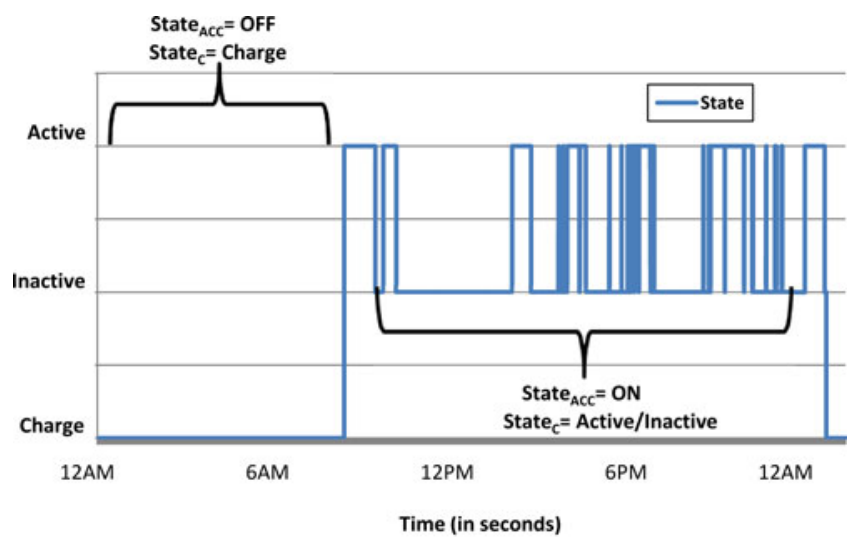

(a)

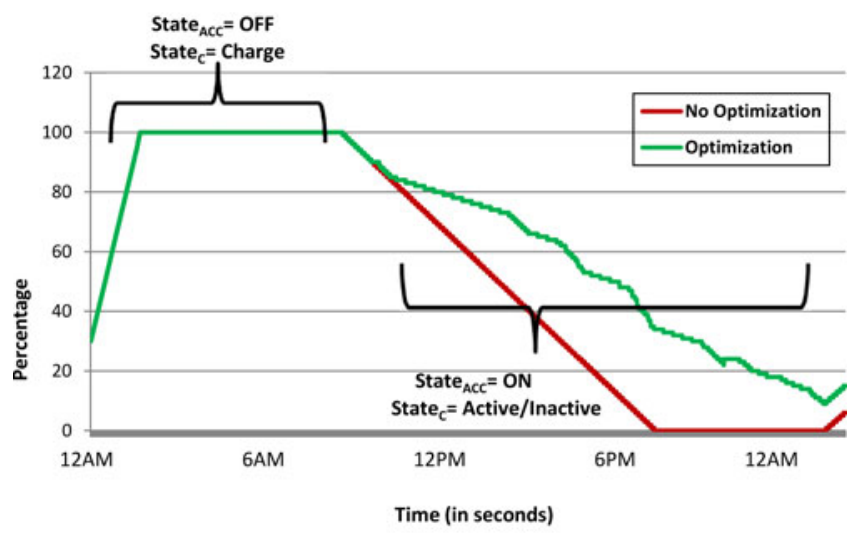

(b)

Fig. 5. First graph (a) shows the data of one of the subjects in the study. The subject charges the phone at $12 \mathrm{am}$, and takes it off the charger and wears the phone at around $8 \mathrm{am}$. Then the subject transitions throughout the day from active to an inactive state. Second graph (b) shows the battery lifetime of the subject in (a). The red line represents an estimated battery lifetime without battery optimization (showing the phone shutting down at 7 P.M., which would prevent the system from capturing the remaining activity performed by the subject. At around 1 A.M., the subject charges the phone again. (a) Sample subject activity. (b) Sample subject battery lifetime.

mode represents the user that carries the smartphone all day in a pouch around their waist and where signal strengths can fluctuate with intermittent $\mathrm{Wi}-\mathrm{Fi}$ and NG communication. Geo-active mode participants in the inlab setting did not use other features such as talk-time, camera, browsing, and gaming, but we acknowledge that in reality such limitations do not exist, so we also tested our system in a real world setting via the Women's Heart Health study.

\section{RESULTS AND DISCUSSION}

A) Battery Lifetime: Battery lifetime is defined as the hours for the smartphone to go from $100 \%$ battery to the ACTION SHUTDOWN event (when the battery is dead and the phone shuts down). Fig. 5 provides a sample of an active subject's state over $24 \mathrm{~h}$, comparing the battery lifetime with and without battery optimization. We can see that without battery optimization the phone shuts down after approximately $9 \mathrm{~h}$, while with battery optimization the phone still has over $34 \%$ battery lifetime remaining at that point. The benefits of battery optimization will be much greater for an inactive participant. 


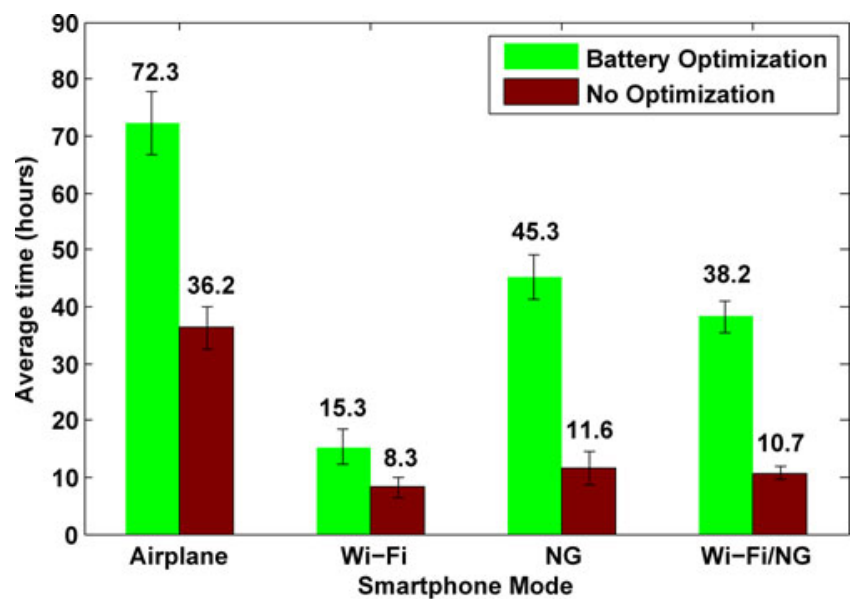

(a)

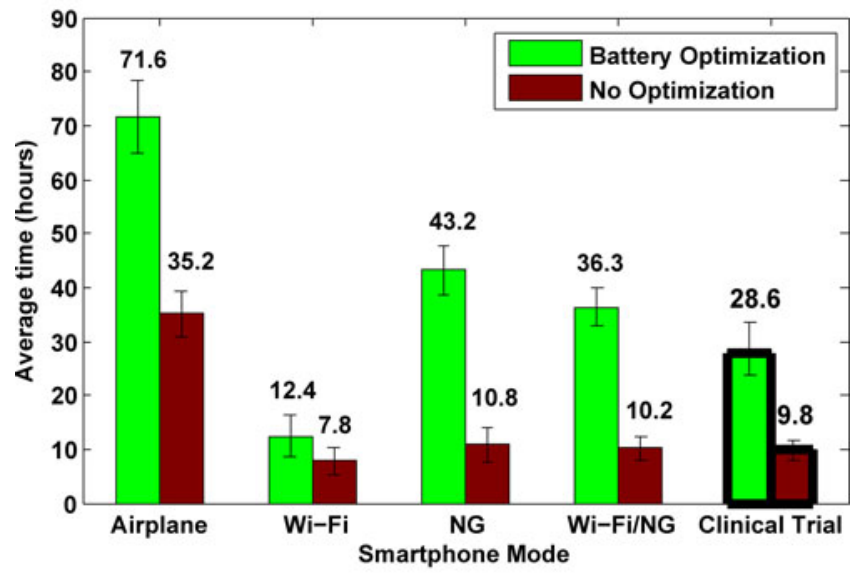

(b)

Fig. 6. Battery lifetime optimization in Geo-inactive and Geo-active mode for the pilot study and Women's Heart Health clinical trial. We notice substantial improvement in battery lifetime with battery optimization in all smartphone settings: Airplane mode, Wi-Fi only mode, NG and Wi-Fi mode, and only NG mode. Results from the Clinical trial are provided that show substantial improvement in battery lifetime by approximately $192 \%$. (a) Geo-inactive mode. (b) Geo-active mode.

Delaying the processing and communication of PA in our battery optimization technique has drastically improved smartphone battery lifetime. We show results for Geo-inactive smartphone settings in Fig. 6(a), averaged over five participants, where we compare results with and without battery optimization. When running the smartphone in airplane mode, we notice $100 \%$ increase, from 36.2 to $72.1 \mathrm{~h}$, almost doubling the lifetime of the battery. Testing in airplane mode purely tests the delay in processing the accelerometer data, as no communication delay can be tested. Increasing the latency in calculations and processing the data in batches results in minimizing file input/output, which in effect reduces computing power.

In Fig. 6(a), we also see improvements in all modes. In the Wi-Fi, NG, and Wi-Fi/NG mode, the battery lifetime increased by $84 \%, 291 \%$, and $257 \%$, respectively. Our results also show that running the system in purely $\mathrm{Wi}-\mathrm{Fi}$ mode is power intensive, lasting only $15.3 \mathrm{~h}$, while running in NG-only mode lasts $45.3 \mathrm{~h}$, almost $192 \%$ that of Wi-Fi mode. When turning Wi-Fi and NG on together, we notice a slight decrease in battery lifetime from $\mathrm{Wi}-\mathrm{Fi} / \mathrm{NG}$ mode to about $38.2 \mathrm{~h}$, with the understanding that

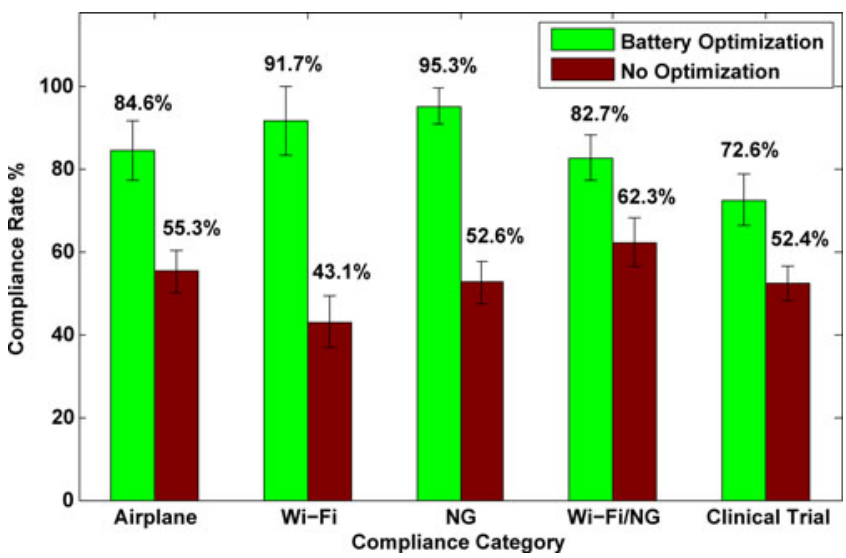

Fig. 7. Comparing compliance rates between the system with and without battery optimization based on each category. We see substantial improvement in compliance in all categories: Activity = physical activity, $\mathrm{DQ}=$ daily questionnaire, $\mathrm{WQ}=$ weekly questionnaire, and $\mathrm{BP}=\mathrm{BP}$ measurements. The overall accuracy shows a $53 \%$ improvement from $55.3 \%$ compliance to $84.6 \%$.

more energy is consumed whenever the smartphone searches for signals.

While Geo-inactive mode showed promise, it is critical to test when participants are actively moving around in areas where there is limited coverage. In Geo-active mode, our results also show strong improvement. Fig. 6(b) compares results with/without battery optimization for both the pilot study and the Women's Heart Health clinical trial.

In airplane mode, with no battery optimization, our system lasted on average $35.2 \mathrm{~h}$, whereas with the optimization battery lifetime doubles to $71.6 \mathrm{~h}$, as in Geo-inactive mode. This can be explained as the smartphone in airplane mode will not be affected by variations in signal strength. From the first inlab setting of Geo-active mode, with no optimization, the results do not exceed $11 \mathrm{~h}$ of battery lifetime in any nonairplane mode [see Fig. 6(b)]. This is concerning as participants would have to charge the phone before the end of the day. In the second in-lab setting, with battery optimization, we see that we are able to achieve improvements of 59\%, 300\%, and $256 \%$ in Wi-Fi, NG, and $\mathrm{Wi}-\mathrm{Fi} / \mathrm{NG}$ modes, respectively.

In a more realistic clinical trial setting, the Women's Heart Health study, we see that on average the participants were able to achieve a battery lifetime of $28.6 \mathrm{~h}$ compared to $9.8 \mathrm{~h}$ without battery optimization. The battery lifetime results in the clinical trial were less than our in-lab setting for Wi-Fi/NG mode, due to the fact that some participants have Wi-Fi turned ON, but also because we do not control their phone usage in terms of browsing, camera time, downloading applications, and playing games. Participants were quite happy with battery longevity, with some realizing that if they forgot to charge their phone at night, their phone battery did not go down. By increasing our expected battery life to $28.6 \mathrm{~h}$, we are able to ensure that most participants will be able to complete a full day with the ease of charging the phone at night.

B) Compliance: Participant adherence to the study protocol is a very critical matter in order to ensure that a remote health monitoring system can be successfully deployed in a real world environment. Fig. 7 shows our battery optimization technique 
resulting in a 53\% increase in overall compliance from $55.3 \%$ to $84.6 \%$

The effects are even more evident in the compliance of PA, where compliance increased from $43.1 \%$ to $91.7 \%$. This result is expected due to the fact that battery lifetime was not lasting the entire day, and many of the participants were complaining that they would have to charge the device mid-day. Many of the participants found the battery life without the battery optimization to be a main concern, and also found that the phone would overheat from the continuous data processing and transmission. Participant response to the DQ showed significant improvement from $52.6 \%$ to $95.3 \%$. The WQ compliance increased with battery optimization due to the fact that a few of the participants would perform their response to the questions during the evening, and when battery drains and is left to charge, the questionnaire would go unanswered. The same effect is shown in the BP measurement compliance rate in Fig. 7.

It is interesting to note that compliance of BP measurements is significantly lower than the other categories, as system messages reminding users to take their BP increased. Factors that enter into noncompliance could be that these women have children and are very busy, but are taught to relax for $5 \mathrm{~min}$ prior to taking their BP measurement. Further investigation is required to see whether the BP monitor was difficult to use, or whether participants had difficulty finding the time and a quiet place to take and transmit the $\mathrm{BP}$ reading using both the phone and the device.

\section{CONCLUSION AND FUtURE WORK}

In this paper, we provide a battery optimization technique to reduce smartphone battery consumption and show the high correlation between battery lifetime and compliance in a remote health monitoring system that uses a smartphone as an information gateway. The WANDA-CVD system has shown success both in lab and real-world settings. Such a system can help reduce battery lifetime for RHM systems that use smartphones to monitor PA. We have shown an ability to drastically enhance compliance by optimizing the battery lifetime of the smartphone application gateway by delaying processing and communication until the phone is charged, resulting in 192\% increase in battery lifetime, yielding on average a 28.6-h battery lifetime in the Women's Heart Health study. The simplicity and ease-of-use of our WANDA-CVD system yielded $85 \%$ overall compliance by participants, and a $95 \%$ compliance rate in PA. Our battery optimization improved compliance by $53 \%$.

The final results from the goals of the clinical trial of the Women's Heart Health study will be published in a future report. In future work, we plan to continue to enhance the battery lifetime of the system, while testing the smartphone application on other devices and under different settings. We also would like to expand the WANDA-CVD system to perform real-time activity classification.

\section{REFERENCES}

[1] (2009). Centers for Disease Control and Prevention. Chronic disease the power to prevent, the call to control. chronfic.pdf. [Online]. Available: http://www.cdc.gov/chronicdisease/resources/publications/AAG/pdf/

[2] R. A. Clark, S. C. Inglis, F. A. McAlister, J. G. F. Cleland, and S. Stewart, "Telemonitoring or structured telephone support programmes for patients with chronic heart failure: Systematic review and meta-analysis," $B M J$, vol. 334, no. 7600, p. 942, May 2007.
[3] C. Worringham, A. Rojek, and I. Stewart. (2011). Development and feasibility of a smartphone, ECG and GPS based system for remotely monitoring exercise in cardiac rehabilitation. PLOS ONE. [Online]. 6(2). Available: http://dx.doi.org/10.1371

[4] N. Alshurafa, W. Xu, J. J. Liu, M.-C. Huang, B. Mortazavi, M. Sarrafzadeh, and C. K. Roberts, "Robust human intensity-varying activity recognition using stochastic approximation in wearable sensors," in Proc. Body Sensor Netw., 2013, pp. 1-6.

[5] N. Alshurafa, J.-A. Eastwood, M. Pourhomayoun, S. Nyamathi, L. Bao, B. Mortazavi, and M. Sarrafzadeh, "Anti-cheating: Detecting self-inflicted and impersonator cheaters for remote health monitoring systems with wearable sensors," in Proc. Body Sensor Netw., 2014, pp. 1-6.

[6] N. Alshurafa, W. Xu, J. Liu, M. Huang, B. Mortazavi, C. Roberts, and M. Sarrafzadeh, "Designing a robust activity recognition framework for health and exergaming using wearable sensors," IEEE J. Biomed. Health Informat., 2013, to be published.

[7] M. K. Suh, C. A. Chen, J. Woodbridge, M. K. Tu, J. I. Kim, A. Nahapetian, L. S. Evangelista, and M. Sarrafzadeh, "A remote patient monitoring system for congestive heart failure," J. Med. Syst., vol. 35, no. 5, pp. 1165-1179, Oct. 2011

[8] N. Alshurafa, J.-A. Eastwood, S. Nyamathi, W. Xu, J. J. Liu, M. Pourhomayoun, H. Ghasemzadeh, and M. Sarrafzadeh, "Battery optimization in smartphones for remote health monitoring systems to enhance user adherence," presented at the Proc. 7th Int. Conf. Pervasive Technol. Relat. Assistive Environ., Rhodes Island, Greece, 2014.

[9] A. S. Desai, and L. W. Stevenson, "Connecting the circle from home to heart-failure disease management," New Eng. J. Med., vol. 363, no. 24, pp. 2364-2367, Dec. 2010.

[10] K. Hung, and Y.-T. Zhang, "Implementation of a wap-based telemedicine system for patient monitoring," IEEE Trans. Inf. Technol. Biomed., vol. 7, no. 2, pp. 101-107, Jun. 2003.

[11] A. G. Logan, W. J. McIsaac, A. Tisler, M. J. Irvine, A. Saunders, A. Dunai, C. A. Rizo, D. S. Feig, M. Hamill, M. Trudel, and J. A. Cafazzo, "Mobile phone-based remote patient monitoring system for management of hypertension in diabetic patients," Amer. J. Hypertens., vol. 20, no. 9, pp. 942-948, Sep. 2007.

[12] I. Kouris, S. Mougiakakou, L. Scarnato, D. Iliopoulou, P. Diem, A. Vazeou, and D. Koutsouris, "Mobile phone technologies and advanced data analysis towards the enhancement of diabetes self-management," Int. J. Electron. Healthcare, vol. 5, no. 4, pp. 386-402, 2010.

[13] A. R. Chowdhury, B. Falchuk, and A. Misra, "Medially: A provenanceaware remote health monitoring middleware," in Proc. IEEE Int. Conf. Pervasive Comput. Commun., 2010, pp. 125-134.

[14] A. Misra and L. Lim, "Optimizing sensor data acquisition for energyefficient smartphone-based continuous event processing," in Proc. IEEE 12th Int. Conf. Mobile Data Manage., Jun. 2011, vol. 1, pp. 88-97.

[15] A. Carroll and G. Heiser, "An analysis of power consumption in a smartphone," in Proc. USENIX Conf. USENIX Annu. Tech. Conf., 2010 pp. 21-21.

[16] Y. G. Lee, W. S. Jeong, and G. Yoon, "Smartphone-based mobile health monitoring," Telemed. J. E Health, vol. 18, no. 8, pp. 585-590, Oct. 2012.

[17] S. I. Chaudhry, B. Barton, J. Mattera, J. Spertus, and H. M. Krumholz, "Randomized trial of telemonitoring to improve heart failure outcomes (Tele-HF): Study design,” J. Card. Fail., vol. 13, no. 9, pp. 709-714, Nov. 2007.

[18] A. Mortara, G. D. Pinna, P. Johnson, R. Maestri, S. Capomolla, M. T. La Rovere, P. Ponikowski, L. Tavazzi, and P. Sleight, "Home telemonitoring in heart failure patients: The HHH study (home or hospital in heart failure)," Eur. J. Heart Fail., vol. 11, no. 3, pp. 312-318, Mar. 2009.

[19] (2013). Healthy people 2020 topics \& objectives. [Online]. Available: http://www.healthypeople.gov/2020/topicsobjectives2020/default.aspx

[20] (2011). Fora care: Healthcare devices. [Online]. Available: http://www. foracare.com/products.html

[21] M. Lan, L. Samy, N. Alshurafa, M.-K. Suh, H. Ghasemzadeh, A. Macabasco-O'Connell, and M. Sarrafzadeh, "Wanda: An end-to-end remote health monitoring and analytics system for heart failure patients," presented at the Wireless Health Conf., New York, NY, USA, 2012.

[22] D. Sanderson, Programming Google App Engine: Build and Run Scalable Web Apps on Google's Infrastructure, 1st ed. Sebastopol, CA, USA: O'Reilly Media, 2009.

[23] Y. Yamada, K. Yokoyama, R. Noriyasu, T. Osaki, T. Adac hi, A. Itoi, Y. Naito, T. Morimoto, M. Kimura, and S. Oda, "Light-intensity activities are important for estimating physical activity energy expenditure using uniaxial and triaxial accelerometers," Eur. J. Appl. Physiol., vol. 105, no. 1, pp. 141-152, Jan. 2009.

Authors' photographs and biographies not available at the time of publication. 